\title{
COMMISSION 26: DOUBLE AND MULTIPLE STARS (ETOILES DOUBLES ET MULTIPLES)
}

Report of Meetings Held at the $21^{\text {st }}$ General Assembly

PRESIDENT: Harold A. McAlister

VICE-PRESIDENT: Helmut A. Abt

\section{INTRODUCTION}

The program for Commission 26 in Buenos Aires consisted of opening and closing business sessions on 24 and 27 July 1991. During two scientific sessions on $26 \mathrm{July}$, the following papers were presented:

A Catalogue of Variable Components in Visual Double \& Multiple Stars

Patricia Lampens, Observatoire Royal de Belgique

Status of the Washington Double Star Catalog

Charles Worley, U.S. Naval Observatory

The CHARA Speckle Programs

Harold McAlister, Georgia State University

The U.S.N.O. Speckle Interferometry Program

Charles Worley, U.S. Naval Observatory

Lunar Occultations and the Hyades

Deane Peterson, S.U.N.Y. Stony Brook

CCD Photometry of Possible Trapezium Systems

Helmut Abt, Kitt Peak National Observatory

Near-IR Studies of Young Binary Stars: Results from Direct Imaging, Speckle Interferometry, and Lunar Occultations

Hans Zinnecker, Universität Würzburg

Double Star Astronomy with the Hubble Space Telescope

Otto Franz, Lowell Observatory

The Commission also participated with Commissions 8 and 24 in the Joint Commission Meeting on Astrometry in the Southern Hemisphere.

\section{SCIENTIFIC SESSIONS}

Approximately 50 people were present during the Commission's scientifc sessions. Authors of the papers presented to the Commission have provided the following summaries of their works.

A Catalogue of Variable Components in Visual Double 8 Multiple Stars - Patricia Lampens, Observatoire Royal de Belgique - A machine readable catalogue of variable components in visual double and multiple stars has been compiled by 
intersecting two recent catalogues, the "General Catalogue of Variable Stars" by P.N. Kholopov et al. (Vols I-III, 4th ed., Moscow, Nauka, 1987) for the variabliity information and the "Catalogue of Components of Double and Multiple Stars" by J. Dommanget (Cont. Van Vleck Obs., vol. 8, pp. 77-82, 1989) for the astrometric information. 468 variable components have been identified. Accurate positions $\left(\sigma_{\alpha}= \pm 0: 01 ; \sigma_{\delta}= \pm 0^{\prime \prime} 1\right)$, component designations and additional identifiers such as $\mathrm{HD}$ and $\mathrm{DM}$ numbers are provided. Relative positions are also given since they are helpful in correctly recognizing the variable component at the telescope. The study of these components in physical double stars is of particular relevance in the fields of stellar structure and evolution.

Status of the Washington Double Star Catalog - Charles Worley, U.S. Naval Observatory - The WDS consists of two compilations of data, plus various ancillary tables and inverse files. The Observation Catalog now contains approximately 433,000 means. It is complete for data published since 1927. For many years, work has proceeded to access the earlier observations, of which 153,000 are now present (estimated completion of 85-90\%). The Index Catalog now contains over 77,000 pairs and has been reformatted to better accomodate modern needs. A new tape version should be available in a few years.

The CHARA Speckle Programs - Harold A. McAlister, Georgia State University The GSU/CHARA speckle program was initiated in 1977 and since 1980 has been carried out at the Kitt Peak 4-m telescope using a remotely controlled speckle camera system employing an intensified CCD array. Participants in this program include W.I. Hartkopf, W.G. Bagnuolo, and the author (all from GSU) and O.G. Franz (Lowell Observatory). The emphasis has been the continuing collection of precise, high resolution measurements of visual, spectroscopic, occultation, and composite spectrum binaries. The CHARA program has now published some 13,000 measures and discovered $\sim 250$ new binary stars. Methods have been developed for real-time data processing to obtain astrometric information, including the elimination of the $180^{\circ}$ quadrant ambiguity inherent in classical speckle interferometry. An algorithm for extracting differential photometric information from speckle data is also near maturation. Since 1988, the CHARA speckle program has been expanded to all-sky coverage with twice yearly trips to the CTIO 4-m telescope.

The U.S.N.O. Speckle Interferometry Program - Charles Worley, U.S. Naval Observatory - In October, 1990, the U.S. Naval Observatory received a new speckle camera system based on an improved version of the successful Georgia State University instrument. With efficient software supplied by the GSU/CHARA group, this system has already made well over a thousand measures at the Washington 26-inch refractor. We would like to emphasize that this instrument arrived aheadof-schedule, below budget, and worked well at the first instance of its use.

Lunar Occultations and the Hyades - Deane Peterson, S.U.N.Y. Stony Brook Obtaining and using lunar occultation data is at once a powerful and very limited technique. Only a fraction of the sky is covered by the Moon's disk during its 
18-yr cycle, but in this region objects can be usefully observed down to about 10 th magnitude at good sites. For binary systems, differential photometry can be obtained (to $\pm 0.1 \mathrm{mag}$ ) along with projected separations (to \pm 1 mas) and angular diameters (to \pm 1 mas for $d \geq 2$ mas). We discuss four Hyades objects for which we have observed, or have tried to observe, occultations, and the impact those data have had on our understanding of the systems and the cluster.

The first of these was Finsen 342, a 13-yr system noticable as a large residual in McClure's solution for the cluster distance. (McClure's technique involves forcing the eclipsing binary, vB22, onto the mass-luminosity relation defined by the visual binaries thereby providing a distance estimate). The occultation trace showed the secondary on the wrong side, leading to a proposal (see Peterson and Solensky 1987 for details) that a 6 -yr period was appropriate. This has since received strong support from speckle observations.

The second system, 51 Tau, was shown by Peterson and Solensky (1988) to serve as a replacement for vB22 in establishing the distance scale. More importantly, it could be added to vB22 and the visual binaries in establishing an even higher weight solution for the distance, the $\Delta \mathrm{m}$ being the major remaining uncertainty. The system escaped observation in the late 1980's, and will do so again in the early 1990's unless observations can be made from the southern hemisphere.

The third system, $\theta^{2}$ Tauri, has been known to be a spectroscopic binary (SB1) for over 80 years, but it was not until the Moon's passages over the cluster center around 1980 that it was discovered that the secondary is only about $1.1 \mathrm{mag}$ fainter than the primary. This lead us to search for the secondary spectrum using high dispersion plates and cross-correlation techniques. The detection of the secondary spectrum was reported by us last year (1990 ASP Conf. on Clusters). Since then the Mark III interferometer has resolved the system and, with the mass ratio, has found the mass and distance to the system. Beyond that, the pair is important in that it consists of a main sequence star and a turnoff star of the same color and a known magnitude difference, which provides a much more stable way of using theoretical isochrones to evaluate the age of the cluster.

The last system, $\theta^{1}$ Tauri, has a potentially significant contribution. The primary is a $\mathrm{K}$ giant and the secondary, $\sim 3.5$ magnitudes fainter and a late $\mathrm{F}$ dwarf, was detected during a series of occultations in the early 1980's from which an angular diameter was also determined for the primary. The system has since been resolved by speckle techniques, and the motion of the primary is being followed by the radial velocity observers as well. By using the measured $\Delta \mathrm{m}$ and colors, placing the secondary on the cluster main sequence will establish the distance. Both the orbital solutions and our knowledge of the mass of the cluster turnoff will provide the mass of the primary. The angular diameter will give the radius, and the characteristics of the cluster provide the age and the initial composition. The only other convective star whose parameters are known to this extent is the Sun. This will provide an important check on the assumption that the mixing length ratio used in interior calculations is the same for all phases of evolution. 
CCD Observations of Possible Trapezium Systems - Helmut Abt, Kitt Peak National Observatory, and Christopher Corbally, S.J., Vatican Observatory Research Group - Trapezium systems are dynamically unstable and should therefore be young. Ambartsumian and Sharpless found them in HII regions, but what are their lifetimes? Allen, Tapia, and Parrao (Rev. Mex. Astr. Ap., vol. 3, p. 119, 1977) scanned 20,000 multiple-star sytems in the IDS and listed 968 apparent Trapezium systems that seemed to be free of optical companions. But they found that the most frequent primary type is $F$, which is not that of young stars.

We obtained CCD observations for 268 of those systems with a 1-m Kitt Peak telescope. They yielded an accuracy in Johnson UBV photometry of $0.06 \mathrm{mag}$ or better. Surprisingly, the mean astrometric accuracy is 0.06 in position angle and $0^{\prime \prime} 07$ in angular separation. But we also needed one MK spectral type per system to determine the distance and reddening. Types have been obtained for 62 systems. Of those, 57 have enough optical components that they are not Trapezium systems. The five remaining Trapezium systems have primary types of O5-B1, so the maximum age of a Trapezium system is about $10^{7}$ years.

Near-IR Studies of Young Binary Stars: Results from Direct Imaging, Speckle Interferometry, and Lunar Occultations - Hans Zinnecker, Universität Würzburg - A selection of recent near-infrared (NIR) results on young, low-mass, spatially resolved binary stars with separations in the range of $0^{\prime \prime} .1$ to $10^{\prime \prime}$ is reported. These results are based on collaborative work with Leinert and Haas (MPIA/ Heidelberg), Christou and Ridgway (NOAO/Tucson), Richichi (Arcetri), and Moneti (ESO/Chile). NIR studies, which are differential due to the coeval nature of the components of young binary systems, bear on binary star formation and on pre-main sequence stellar and circumstellar evolution. From direct NIR array imaging of known T Tauri binaries, we found (A\&A, vol. 242, p. 428) that the stronger NIR color excess tends to reside with the more luminous component at $2 \mu$, possibly implying active accretion disks to be present around each individual stellar component or preferentially around the brighter component. With slit scanning (1D) speckle interferometry we detected XZ Tau to be a binary with projected separation of 0".3 (A\&A vol. 230, p. L1). The infrared companion of XZ Tau brings the roster of $\mathrm{T}$ Tauri stars with infrared companions to 5 (the others being T Tau, Haro 6-10, Glass I, and Elias 22). By the same means we also identified GG Tau as a hierarchical quadruple system. Thus, speckle data have shown that both GG Tau and GG Tau/c (10" south) are themselves binaries ( $0^{\prime \prime} .3$ and $1^{\prime \prime} .4$ apart, respectively). Using a NIR speckle camera, we investigated the known binaries S CrA, AS 205, and T Tau in 2D for the first time, and discovered the duplicity of Elias 49 (MWC 863), DD Tau, FX Tau, and $\mathrm{LkCa}$ 3. We confirmed the FU Ori object $\mathrm{Z} \mathrm{CMa} \mathrm{to} \mathrm{be} \mathrm{a} \mathrm{binary} \mathrm{with} \mathrm{a} \mathrm{projected}$ separation of $0^{\prime \prime} .10$ (following private communication by Beckwith) and suggested that tidal interaction might trigger the FU Ori outburst in the circumstellar disk of either the primary or the secondary. Finally, lunar occultations at $2 \mu$ (similar to those pioneered by Simon et al.) confirmed the binary nature of FV and FW Tau. However, no spatially extended NIR emission around the individual components in 
these systems could be detected, contrary to the single T Tauri star DG Tau where we have seen a halo of size 45 mas or about $6 \mathrm{AU}$, the smallest structure resolved up to now in any young star.

Double Star Astronomy with the Hubble Space Telescope - Otto Franz, Lowell Observatory - An orbital solution from visual micrometry measures necessarily made mostly near apastron for the binary star ADS $11300=\mathrm{Hu} 581=$ WDS $18229+1458$ predicted periastron passage in 1984 at a separation not resolvable by visual observers. Realizing that a single high resolution observation at the present time would provide crucial information to define the orbit, ADS 11300 was observed with the Hubble Space Telescope Fine Guidance Sensors on 1 November 1990. The data yielded an angular separation of $0^{\prime \prime}: 066$. This new result permitted a revised prediction of periastron passage during 1992 at a separation below 0".01, making ADS 11300 a challenging target for continued HST-FGS astrometric measurement.

\section{COMMISSION BUSINESS}

During the first business session, the President welcomed those in attendance and asked for a moment of silence in memory of two Commission members who passed away since the last General Assembly, Wallace R. Beardsley and Raymond H. Wilson.

A brief discussion was conducted concerning the initiative from the General Secretary during the triennium to amalgamate certain commissions which appeared to have common scientific ties. It was the strong consensus of all commissions concerned not to amalgamate. It was recognized that a reassessment of commission structure was appropriate from time to time, but that such reassessments should be based solely upon scientific grounds and be carried out with extensive input from the membership.

The results of the election of new officers were announced - those results being:

President: H. Abt (USA) Vice President: C. Worley (USA)

Organizing Committee - New Members: Y. Balega (USSR), F. Fekel (USA), C. Scarfe (Canada), and H. Zinnecker (Germany); Continuing Members: P. Bernacca (Italy), H. McAlister (USA), and E. Van Dessel (Belgium).

On behalf of a letter written by J. Dommanget (Belgium) to the Commission President and distributed to other astronomers as well, K.Aa. Strand (USA) brought up the question of accessibility of the Washington Double Star Catalogue (WDS) to the scientific community. The President responded that this was not appropriately Commission business because, whereas the Commission has always strongly supported the efforts involved in maintaining the WDS and its predecessor catalogs, the WDS was a scientific effort carried out and funded by the U.S. Naval Observatory. The USNO Scientific Director G. Westerhout reiterated this point and emphasized that the data within the WDS are routinely and promptly provided to any requesting astronomer without charge as a service of the USNO. 
C. Worley explained the impracticality of maintaining the WDS data base, which is essentially not a complete collection and hence is being continuously revised, at the various data centers. This argument was strongly seconded by W. Warren of the Astronomical Data Center at Goddard SFC.

Upon the recommendation of R.S. Harrington (U.S. Naval Observatory), we remind the orbit computers of the need for more accurate ephemeris calculations to support the new observing techniques becoming available. There will soon be the need to allow for variations of elements in many systems, and in one or two cases even now. These variations may be due to change in perspective due to intrinsic motion or to dynamical variations in multiple systems. To this end, parameters that must be included are the epoch of orientation of the node and the epoch of osculation of the orbit. If the epoch of osculation is not available, give at least the weighted mean epoch if appropriate. We recommend starting these practices now so that they will be standard when required.

Vice President Abt chaired the final business session. New members of the IAU who selected Commission 26 for their affiliation were announced by $\mathrm{H}$. McAlister to be: Yuri Balega (USSR), Silva Gaudenzi (Italy), Jon E. Hakkila (USA), Patricia Lampens (Belgium), Roberto Morbidelli (Italy), Corinne Rossi (Italy), Dimitrios Sinachopoulos (Belgium), and James R. Sowell (USA). Astronomers already holding IAU membership who newly wished to join the Commission were: John Davis (Australia), Hartmut Jahreiss (Germany), and Deane Peterson (USA). These additions to the Commission bring the total membership to 109 astronomers.

As at the last General Assembly, the future of the Commission's Circulaire d'Information was discussed. The Circulaire has been published at their expense by Drs. Muller and Couteau at the Observatoire de Nice, an activity much appreciated by the Commission and by the entire double star community. It is hoped that this publication can continue and its use be expanded.

$\mathrm{H}$. Abt conducted a thought provoking discussion of the status of various subfields of double star astronomy including astrometry (positions and parallaxes), visual micrometry, interferometry, occultation studies, photometry, radial velocities, spectroscopy (MK types and abundances), evolutionary studies, and dynamical studies. The most mature of these areas was considered to be the several aspects of theoretical dynamics while virtually every other area was far from saturated by both results and potential contributers.

The question of "standard stars", or more precisely systems commonly observed by groups employing different observational techniques for measuring $(\theta, \rho)$, was raised by $C$. Worley. This is particularly important for speckle and other types of interferometry where spatial calibration may not be straightforward and for space observations from HIPPARCOS and HST. It was agreed that a committee consisting of O.G. Franz, K. Johnston, H. McAlister, and D. Peterson would work toward the goal of suggesting a list of "standards" along the lines of a previous attempt by McAlister and W.I. Hartkopf (P.A.S.P., vol. 95, p. 778, 1983). 